\title{
EUS - experimental and evolving techniques
}

A. Fritscher-Ravens

Dep. Gastroenterology, Homerton University Hospital, London E9 6SR, UK

In recent years both the utility and the technique of endoscopic ultrasound (EUS) have become more established in the minds of physicians. It has also become more widely available as more

Correspondence: A. Fritscher-Ravens · Dep. Gastroenterology · Homerton University Hospital · London E9 6SR, UK

Bibliography: Endoscopy 2006; 38 (S1): S95-S99 @ Georg Thieme Verlag KG Stuttgart · New York · ISSN 0013-726X · DOI 10.1055/s-2006-946666 
medical centres have added it to their diagnostic repertoire. The development of echoendoscopes with larger accessory channels has opened up the possibility for further developments of new devices and procedures. Needles with larger diameter have become available, which besides obtaining tissue from the target organ, may now also allow the injection of agents or objects into it $[1,2]$. These new developments have laid the ground for the evolution of an exiting new dimension of use: EUS-guided surgical therapy.

As much as minimally invasive surgery has resulted in reduced scaring, shorter hospital stays and a favourable cost-benefit ratio compared to conventional surgery, the trend to even further minimize the surgical approach has lead to new, transgastric endoscopic procedures without any percutaneous access to the abdominal cavity [3-9].

Parallel to this new natural orifice transgastric endoscopic approach (NOTES) (10) the EUS-train has moved on to explore new territory of more invasive therapeutic use, where EUS serves as a guide for the transgastric assessment of organs within the peritoneal cavity without the need of direct access [11 - 14]. But even if direct access was necessary, EUS can select pathology because of its unique ability to explore and visualize the inside of organs without direct viewing. This ability can be used to find and guide as where and which lesion should be endoscopically operated upon. This overview will describe some of the possible future EUS-guided techniques, which might evolve into routine procedures as soon as the necessary basic tools become commercially available.

\section{Tissue apposition and sewing}

The perception of just being able to view gastrointestinal organs and to needle them to obtain biopsy samples is progressing towards more invasive, therapeutic EUS-guided interventions. Since EUS allows visual access to organs adjacent to the GI tract such as mediastinal and paragastric lymph nodes, the gall bladder, small bowel or parts of the diaphragm, endoscopic surgical procedures are being developed, to treat conditions that would otherwise require open or laparoscopic surgery. The first step in performing such procedures is to create access through the stomach or any other gastrointestinal tract wall with an EUS needle and then appose the desired organ or structure of the peritoneal or mediastinal cavity to the gut wall. To achieve this and to sew two items together a new and rather simple sewing method has been recently developed [14].

Tissue apposition and sewing is performed using a commercially available flexible, 19 gauge EUS-needle, produced for routine use, into which a custom-made metal anchor attached to a thread can be loaded. The hollow center is normally reserved for the tissue sample after the inner stylet has been removed. In this case the inner stylet needs to be pulled back approximately $2 \mathrm{~cm}$ to provide space for the anchor, and is subsequently used as the ejection tool. The anchor-thread runs along the outside of the EUSneedle within the accessory channel of the echoendoscope.

The loaded needle can be guided from any accessible gut lumen into a structure or organ within reach of the EUS-imaging under real-time visualization. Once the needle has been pushed into the target the needle-stylet can be pushed forward to eject the anchor. The success of this maneuver can be seen on the EUSscreen, as the anchor would appear as a hyper-echoic structure with some reflections. The stylet can be removed from the needle lumen to provide access to the target for additional devices such as guide-wires. Following the ejection the examiner can pull on the anchor-thread from the outside of the endoscope, which will enable the anchor to pull the target organ towards the gut wall. If necessary, the thread can be locked as a stitch against the accessible inner gut wall or be kept outside the patient for further manipulation. Specifically for this device, a locking and cutting mechanism was designed to either lock one thread against a structure or two or more threads together like a stitch. This "suture system" represents a basic kit for the new and more interventional procedures such as EUS guided lymph node removal [15]. It has been developed and refined over the last few years and will probably become commercially available in 2007.

\section{Gastrojejunal anastomosis}

EUS-guided gastrojejunal anastomosis to bridge obstructing malignancies was the first procedure developed some 5 years ago. Under EUS vision the anchor and thread method, described above, was used to capture a small bowel loop exclusively from the inside of the stomach and appose the small bowel to the peritoneal side of the gastric wall [11]. Several methods were tried to provide access for one device to the small bowel and yet another to the gastric lumen in order to compress both the tissues of the small bowel and the gastric wall causing ischaemia with subsequent fistula formation $[11,13]$. The device, which required nondirect access to the lumen of the selected organ, needed to be small enough to be passed over a 0.035 guide wire. A variety of anchor designs were tried including small portions of $5 \mathrm{~F}$ and $7 \mathrm{~F}$ tubing, joined together in such a way, that they would form a cross, once released, a plate and spring inserted from the gastric side, and balloons with epoxy filling, which would harden under infrared light [13]. The best option to date has been a double balloon system, which can be passed over a guide wire, placed into the small bowel lumen through the EUS-needle, into the small bowel lumen [16]. It can then be inflated and filled with water to provide counter pressure from the non-accessible small bowel side against a second balloon, inflated at the gastric side, after the small bowel balloon had been pulled at to provide closest possible apposition of the two balloons and the wall. This will force the two walls (small bowel and stomach) together with a pressure higher than $200 \mathrm{mmHg}$, which in general is high enough to cause ischaemia to the tissue in between. The remaining catheter is cut and the system stays in place for a few days to form an opening between the small bowel and the gastric wall. The anastomosis achieved can be quite small and may need to be further opened. More developments are on the way, as survival animal studies have been successfully performed (11). However, studies in humans are still awaited.

In a porcine model, the same technique has been used for a connection between the gall bladder and the stomach (cholecystogastrostomy) to allow gall-stones to be removed [17]. In humans this 
access could also be used for the drainage of a gall bladder empyema. Other indications, where this double balloon system could be used would include colon-colon anastomosis for inoperable colon cancer or anastomosis in the process of obesity surgery.

\section{EUS guided posterior gastropexy for reflux disease}

Attempts to treat gastroesophageal reflux disease due to hiatus hernia exclusively endoscopically have been made recently. Some procedures such as endoscopic gastroplication [18] have proven to be reasonably successful. Others are only rarely used or have been withdrawn from the market. Traditionally, if medical treatment remains unsuccessful, reflux disease would be treated by surgery (laparoscopically, by laparotomy), which would attempt to repair the hiatus hernia. A variety of surgical techniques have been used of which the fundoplication described by Nissen and that by Hill have given good results, while the Nissen fundoplication is the used in the vast majority of cases in daily routine surgery [19].

Under EUS guidance an endoscopic attempt, which closely relates to and is based on the surgical Hill procedure, could add a less invasive approach to the currently available range of nonsurgical treatment options.

The attempted EUS-guided gastropexy utilizes the suturing system, described earlier $[12,14]$. Two separate stitches using anchors are placed through the gastric/cardia wall with one anchor being ejected into the median arcuate ligament (MAL), and the other just beyond the wall of the lower esophageal sphincter. Synchronous real time EUS imaging enables exact placement of these anchors into the desired muscle or just beyond the gut wall, and serves as the imaging technique to guide the correct placement of tools or devices for the fixation of the lower esophageal sphincter $[12,14]$. After anchor ejection both threads, which are tightly connected to the anchors appear in the gastric and cardia lumen and have to be locked together endoscopically. To achieve the necessary pull-force on the thread fixed to the cardia, manometry has to be performed prior to, during and after the procedure to measure the alterations of the pressure in the LES. This is again performed similar to the surgical Hill procedure. In experimental studies performed in more than 20 survival procedures in small pigs the median sphincter pressure increased significantly from $11.2 \mathrm{mmHg}$ to $21 \mathrm{mmHg}(\mathrm{p}<0.05)$ (12). The median length of the LES increased from 2.8 to $3.5 \mathrm{~cm}$. Even four weeks after the procedure, these data were reproducible. All the studies were performed in a porcine model without hiatus hernia and evidence, that this procedure might be successfully applied in humans is not yet available, as the suturing system has not been approved for patients use to date, but is awaited for 2007.

\section{EUS-guided lymph node removal}

The combination of both, EUS and transgastric manipulations also seems feasible. This approach can be especially useful to assist other endoscopic techniques such as Endoscopic Mucosa Resection (EMR), when lymph node removal seems necessary. If prior to EMR lymph node involvement is suspected, these patients need aggressive surgery. To date, there is no possibility to remove these potentially affected nodes without surgery. Any sentinel nodes are a good example of these occasions. Other reasons to remove an entire, pre-selected lymph node may especially include situations, when prior biopsies were repeatedly inconclusive.

Single lymph nodes may need extirpation in an "attempt to cure" or for diagnostic purposes.

EUS cannot only detect small $(<1 \mathrm{~cm})$ peri-esophageal or/and peri-gastric lymph nodes but can also select "suspicious" nodes by their echo-morphology. In addition, it enables disclosure of possible intervening blood vessel between the gut wall and the node, which then provides a more secure an safe transgastric access. After selection of such a node EUS may also guide the examiner to capture the target with an anchor under real time visualization.

A commercially available 19 gauge EUS needle with the custommade anchor and thread (as described above) can be guided through the full length of the target node and be ejected just beyond it. The T-bar anchor then tilts into a horizontal position to catch the node. The capturing of the lymph node can be seen on the ultrasound screen in real time. When the examiner pulls on the thread the node will be following the thread-path to the gastric wall; when the thread is released, the node bounces back into its original position. When pulled on the thread, it may indent into the gastric lumen [15].

Subsequent endoscopic incision of the gastric wall using a needle knife alongside or over the thread has to be performed under direct endoscopic view. Care has to be taken, as the electrocautery may damage or cut the thread. The gastric incision can be kept very small. After full thickness opening of the gastric wall, the lymph node can be removed by pulling on the thread, which will force the anchor to take the node along with it through the incision into the gastric lumen, and out of the patient. Once the specimen is retrieved, the gastric laceration has to be closed endoscopically. This procedure is performed with the suturing system (anchor and thread), implanted into the hollow part of an EUS needle, which was described above and is the same type anchor and thread that the node was captured with $[14,15]$.

After the anchor has been loaded into a 19 gauge EUS-needle and passed through the accessory channel of the gastroscope, the endoscope has to be brought into close proximity to the gastric incision. The needle has to be guided through the entire gastric wall before the anchor can be deployed. The process needs to be repeated with another anchor deployment on the opposite side of the incision. Once opposing stitches have been placed the threads are locked together by the accompanying endoscopic locking device, which is suitable for the passage through the accessory channel of a norm endoscope. The threads are cut concurrently as they are locking by this device $[4,14]$.

This concept has been proven feasible in a few animal survival studies. Further and larger animal studies are needed before embarking on studies in humans. 
EUS-guided approaches to the heart

The proximity of cardiac structures to the wall of the esophagus might allow a variety of trans-esophageal interventional procedures to be performed under endoscopic ultrasound control. There will be concerns about safety and the risk of bleeding of this moving target. However, if feasible it would open a variety of examinations, which would be less difficult and cumbersome to perform than the current approaches through veins or the USor CT-guided percutaneous route for such procedures as myocardial biopsies or injections. To investigate the feasibility and safety of a variety of interventional trans-esophageal cardiac procedures, experimental studies in five live pigs were undertaken [20]. In addition one biopsy of a lesion of unknown aetiology was obtained from the left atrium of a seriously ill patient.

A variety of conventional and new EUS devices were tested in survival experiments in pigs. These included trans-esophageal intra-cardiac 22 and $19 \mathrm{G}$ needles, guide-wires, radio-frequency needles, electrodes, pacing wires and injection of contrast. Excellent EUS views could be obtained of the left atrium, aortic valve, pulmonary trunk and left coronary artery. It was possible to pace the heart through the EUS scope under direct ECG- and vital function control. Diathermy was applied to the aortic valve and cutting diathermy applied to a needle which had been passed through a leaflet of the aortic valve [20].

It was easy to puncture the heart with both 22 gauge and 19 gauge EUS needles. Using the smaller size needle electrodes ECG traces were obtained directly from the surface of the heart and also from intra-cardiac sites. Experimental procedures studied included needle biopsies, contrast medium injections into the atrium and the coronary arteries, passage of a guide-wire into atrium and ventricle, direct intra-cardiac recording of ECG, cardiac conductive tissue ablation, direct cardiac pacing (21). It was possible to insert a 22 gauge needle into the left coronary artery adjacent to the aortic valve root and to inject contrast into the artery under EUS control. No bleeding or electrical instability was observed in these survival experiments in $30-35 \mathrm{~kg}$ pigs. In 2 patients pericardial fluid was aspirated using the small calibre EUS needle. In 1 patient with an apparent intra-cardial tumour thought to be a myxoma a 19G EUS biopsy was performed without any change of vital functions or ECG during the procedure or afterwards. There were no complications. These preliminary studies suggest that some trans-esophageal intra-cardiac procedures are feasible and that cautious extension of these preliminary experiments and experiences are warranted.

\section{Conclusion}

Recently physicians have been evolved the exiting new experimental field of natural orifice transluminal endoscopic interventions (NOTES) to perform minimally invasive endoscopic surgery.

This new approach has become fashionable and many centers across the world have performed their first animal studies in this respect. However, in humans any possible procedures will have to be compared with traditional surgery in terms of safety and complication rates. Possible complications need especially to be treatable via the endoscopic route.

Parallel to the expansion of endoscopic techniques new indications for therapeutic EUS-guided interventions have been developed in areas that have been purely surgical until now. The EUSguided approach may be of some benefit when compared to the purely endoscopic technique. Although more complicated, it is less invasive and some of the possible complications such as bleeding from larger vessels seem avoidable, as vessels can be detected with EUS prior to being punctured, and thus the attempted route can be changed. Combined solely EUS guided and transgastric approaches such as EUS-guided lymphadenectomy may offer benefits, which purely transgastric endoscopic procedures cannot deliver such as the preselection of a possible malignant node.

Although EUS is only a small niche in endoscopy it may prosper with these new interventions, once gastroenterologists and surgeons become interested in these new techniques and teaching facilities have become available for those, who would like to get involved.

\section{References}

${ }^{1}$ Chang KJ, Nguyen PT, Thompson JA, Kurosaki, Casey LR, Leung EC, Granger GA. Phase I clinical trial of allogeneic mixed lymphocyte culture (cytoimplant) delivered by endoscopic ultrasound-guided fineneedle injection in patients with advanced pancreatic carcinoma. Cancer. 2000; 88: 325-335

${ }^{2}$ Gunter E, Lingenfelser T, Eitelbach F, Muller H, Ell C. EUS-guided ethanol injection for treatment of GI stromal tumor. Gastrointest Endosc 2003; 57: 13 - 115

${ }^{3}$ Kalloo AN, Singh VK, Sanjay B, Jagannath SB, Niiyama H, Hill SL. Flexible transgastric peritoneoscopy: a novel approach to diagnosis and therapeutic intervention in the peritoneal cavity. Gastrointest Endosc 2004; 60: $14-117$

${ }^{4}$ Ikeda K. Fritscher-Ravens A, Mosse CA, Mills T, Tajiri H, Swain CP. Endoscopic full-thickness resection with sutured closure in a porcine model. Gastrointest Endosc. 2005; 62: 122 - 129

${ }^{5}$ Hu B, Chung SCS, Sun LCL, Kawashima K, Yamamoto T, Cotton PB, Gostout CJ, Hawes RH, Kalloo A, Kantsevoy SV, Pasricha PJ. Transoral obesity surgery: Endoluminal gastroplasty with an endoscopic suture device. Endoscopy 2005; 37: 411 - 414

${ }^{6}$ Kantsevoy SV, Hu B, Jagannath SB, Vaughn CA, Beitler DM, Chung SS, Cotton PB, Gostout CJ, Hawes RH, Pasricha PJ, Magee CA, Pipitone LJ, Talamini MA, Kalloo AN. Transgastric endoscopic splenectomy. Is it possible? 2006; 20: $22-25$

${ }^{7}$ Kantsevoy SV, Jagannath SB, Niiyama H, Chung SS, Cotton PB, Gostout CJ, Hawes RH, Pasricha PJ, Magee CA, Vaughn CA, Barlow D, Shimonaka H, Kalloo AN. Endoscopic gastrojejunostomy with survival in a porcine model. 2005; 62: 87-92

${ }^{8}$ Bergstrom M, Ikeda K, Swain P, Park PO. Transgastric anastomosis by using flexible endoscopy in a porcine model. Gastrointest Endosc. 2006; 63: $307-312$

${ }^{9}$ Park PO, Bergstrom M, Ikeda K, Fritscher-Ravens A, Swain P. Experimental studies of transgastric gallbladder surgery: cholecystectomy and cholecystogastric anastomosis. Gastrointest Endosc 2005; 61: 1 -6

${ }^{10}$ Bowman DE. ASGE/SAGES working group on natural orifice translumenal endoscopic surgery: White paper October 2005. Gastrointest Endosc 2006; 63: 199-203

${ }^{11}$ Fritscher-Ravens , Mosse CA, Mukherjee D et al. Translumenal Endosurgery: Single lumen access anastomosis device for flexible endoscopy. Gastrointest Endosc 2003; 58: 585-591

12 Fritscher-Ravens A, Mosse CA, Mukherjee D et al. Transgastric gastropexy and hiatus hernia repair for gastro-esophageal reflux disease under endoscopic ultrasound control: an experimental study in a porcine model. Gastrointest Endosc 2004; 59: 89-95 
${ }^{13}$ Fritscher-Ravens A, Mosse CA, Mukherjee D, Swain CP. Balloon compression anastomosis at flexible endoscopy. Gastrointest Endosc 2003; 57: AB6

${ }^{14}$ Fritscher-Ravens A, Mosse CA, Mills TN, Mukherjee D, Park PO, Swain CP. A through-the-scope device for suturing and tissue approximation under EUS control. Gastrointest Endosc 2002; 56: 737 - 742

${ }^{15}$ Fritscher-Ravens A, Mosse CA, Ikeda K, Swain P. Endoscopic transgastric lymphadenectomy by using EUS for selection and guidance. Gastrointest Endosc. 2006; 63: 302 - 306

${ }^{16}$ Swain CP, Lichtenberg E, Spelde H, Fox B, Park PO, Bergstrom M, Ikeda K, Mosse CA, Fritscher-Ravens A. Double balloon anastomosis: transgastric gastro-jejunostomy. Gastrointest Endsoc, in press

${ }^{17}$ Fritscher-Ravens A, Mosse S, Mills T, Swain P. Endoscopic cholecystogastropexy, cholecystogastrostomy, and gall stone removal under endoscopic ultrasound control. GUT 2004; 53 (suppl. IV): A43

18 Thomson M, Fritscher-Ravens A, Hall S, Afzal N, Ashwood P, Swain CP. Endoluminal gastroplication in children with significant gastro-oesophageal reflux disease. GUT 2004; 53: 1745 - 1750

${ }^{19}$ Low D, Anderson RP, Ilves R, Ricciardelli E, Hill LD. Fifteen- to twentyyear results after the Hill antireflux operation. J Cardiovasc Surg 1989; 98: $444-450$

${ }^{20}$ Fritscher-Ravens A, Mosse AC, Ikeda K, Swain P. Interventional cardiac endoscopic ultrasound. Gastrointest Endosc 2005; 61: AB277 